\title{
Duerochelys arribasi, nov. gen., nov. sp., Pelomedusidae du Ludien du Bassin du Duero (Espagne)
}

\author{
par Emiliano JiMÉneZ FUENTES *
}

\begin{abstract}
Sommaire. - On décrit un nouveau Chélonien de la famille des Pelomedusidac : Duerochelys arribasi, procédant d'un gisement ludien de Aldearrubia (Salamanque, Espagne), connu par deux exemplaires : un plastron incomplet et un entoplastron. Il se caractérise par deux intergulaires, cas uniquo chez les Pelomedusidae.
\end{abstract}

Ces dernières années, de nombreuses découvertes de Chéloniens sont en train d'enrichir les connaissances paléontologiques du Paléogène dans le SW du Bassin du Duero, très imparfaitement connu jusqu'à nos jours.

Au siècle dernier, d'éminents géologues qui se consacrèrent à ce bassin et à sa paléofaune (parmi eux, Ezquerra, Prado, Gervais et Verneuil) datèrent du Miocène la grande masse de sédiments tertiaires ; effectivement, ces matériāux couvrent une grande partie de ce territoire, cependant d'autres géologues (tels que Gil y Maestre, Puig y Larraz, Vilanova, Miquel, Larrazet, etc.) signalèrent la présence au $\mathrm{NE}$ et $\mathrm{SW}$ de sédiments plus anciens, en se basant sur la comparaison stratigraphique du Bassin parisien.

En ce qui concerne le SW de ce bassin au début du siècle, les connaissances paléontologiques se limitaient aux citations du Crocodilus rollinatti à Sanzoles (Zamora) [J. Vilanova, 1873], et du Mastodon

$\quad$ Fossiles
Reptiles indéterminés
Palaeotherium magnum
Palaeotherium minus
Palaeotherium curtum
Reptiles indéterminés
Creodonto ind.
Stereogenys salmanticensis ${ }^{1}$
Stereogenys salmanticensis ${ }^{1}$
Podocnemis carbajosai ${ }^{1}$
Podocnemis entodermica ${ }^{1}$
Allaeochelys casasecai
Crocodilia ind.
Podocnemis sp. ${ }^{1}$

angustidens et Acerotherium incisivum à Corrales (Zamora) [G. Puig y Larraz, 1883].

La première découverte importante date de 1906 ; Miquel découvrit alors le "Paloplotherium " minus et Xiphodon gracile [M. Miquel, 1906], ainsi que quelques Reptiles à San Morales, à $14 \mathrm{~km}$ à $\mathrm{l}$ 'E de Salamanque. Si cela ne permit pas de préciser l'âge du gisement, vu la grande extension verticale de ces fossiles elle la confirma comme étant du Paléogène.

Bien plus importante fut la découverte du Lophiodon isselense et du Chasmotherium minimum à Corrales (Zamora) [F. Roman et J. Royo Gómez, 1922 ; F. Roman, 1923] (première citation, sans spécification [E. Hernández Pacheco, 1915] qui a daté ce gisement à l'étage supérieur du Lutétien moyen.

D'autres découvertes postérieures sont exposées dans le tableau suivant :

$\begin{array}{cl}\text { Localite } & \text { Bibliographie } \\ \text { Aldealengua } & {[27]} \\ \text { San Morales } & {[8]} \\ " & \\ \text { Sanzoles } & " \\ \text { San Morales } & " \\ \text { " } & " \\ \text { Aldealengua } & {[12,13,14,17]} \\ \text { Corrales } & {[12,17]} \\ \text { Aldealengua } & {[15]} \\ \text { " } & {[17]} \\ \text { Corrales } & {[16]} \\ \text { " } & {[16]} \\ \text { Aldearrubia } & {[18]}\end{array}$

\footnotetext{
* Dépt de géologie. Faculté des sciences. Université de Salamanque (Espagne). Note présentée à la séance du 2 décembre 1974. 
Les connaissances stratigraphiques ont subi récemment [E. Jiménez, 1972 ; 1973] de grandes modifications, résultat auquel on aboutit si l'on considère l'énorme importance que l'orogénie alpine a eu dans la région avec une tectonique de failles.

Une de ces probables fractures explique que deux gisements paléontologiques, avec une stratigraphie semblable et à une distance relativement petite, présentent entre eux une dénivellation de plus de $50 \mathrm{~m}$ : ce sont les gisements de "la Aceña de la Fuente " (San Morales) [M. Miquel, 1906 ; E. Hernandez Pacheco, 1943 ; M. Crusafont et J. Truyols, 1957] et daté du Ludien et le "Teso de las Canteras " décrit plus récemment [E. Jiménez, 1972] ; c'est dans ce gisement, situé à $1,5 \mathrm{~km}$ à l'E de Aldearrubia (Salamanque) que M. Eduardo Carbajosa découvrit deux fragments de Chéloniens, ce qui est à l'origine de ce travail. Chronologiquement le deuxième que nous allons décrire fut trouvé en premier, étiqueté dans la collection de la Faculté de Sciences de l'Université de Salamanque (F.C.U.S.) avec le numéro 226. Dès sa découverte, la présence d'un double intergulaire nous a frappé, mais l'incomplète connaissance de ses caractères ne nous permit pas de divulguer alors cette découverte. Peu de temps après, la découverte d'un nouvel exemplaire plus complet dans le même gisement se produisit, celui-ci fut inscrit dans la même collection avec le numéro 326 .

Sa description est la suivante :

DUEROCHELYS ARRIBASI, nov. gen., nov. sp. Holotype et espèce-type : ex. 326 F.C.U.S. (fig. 1 et 2).

Ce spécimen se compose d'un plastron ventral auquel il manque seulement les xiphiplastrons. Le fragment mesure $25 \times 23 \mathrm{~cm}$ et par ses caractéristiques il peut être classé dans la "superfamille " des Pleurodirae. La disposition des écussons et des plaques est semblable à celle de la famille des Pelomedusidae, mais il possède deux intergulaires, ce qui n'existe pas dans cette famille. Il est apparu enlisé dans un grès, compact et rougeâtre.

Le lobe antérieur est arrondi, deux fois plus large que long et légèrement courbé en avant. Il a une surface lisse d'où ressortent les sillons dermiques et les sutures osseuses.

Dans sa face interne, il n'a pas été possible de séparer la matrice sablonneuse, car en le tentant nous avons observé que la matière osseuse était dans cette partie en très mauvais état, à l'exception de l'entoplastron.

Le pont sternal est de $160 \mathrm{~mm}$ de longueur et nous ne lui connaissons que de timides ébauches de plaques marginales.

Écussons épidermiques. L'animal possédait deux intergulaires triangulaires et en contact par la ligne médiane. Ils touchent les gulaires en les séparant, ainsi que les huméraux. Les sillons avec ceux-ci sont légèrement courbés.

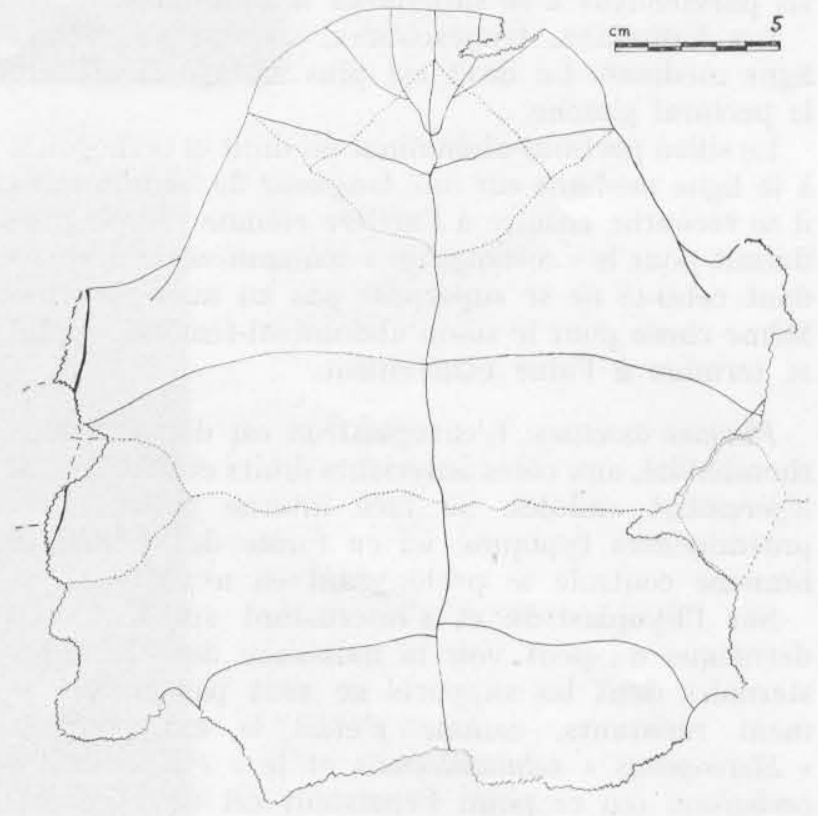

FIG. 1. - Duerochelys arribasi nov. gen., nov. sp. (Ex. 326, F. C. U. S.). Schéma.

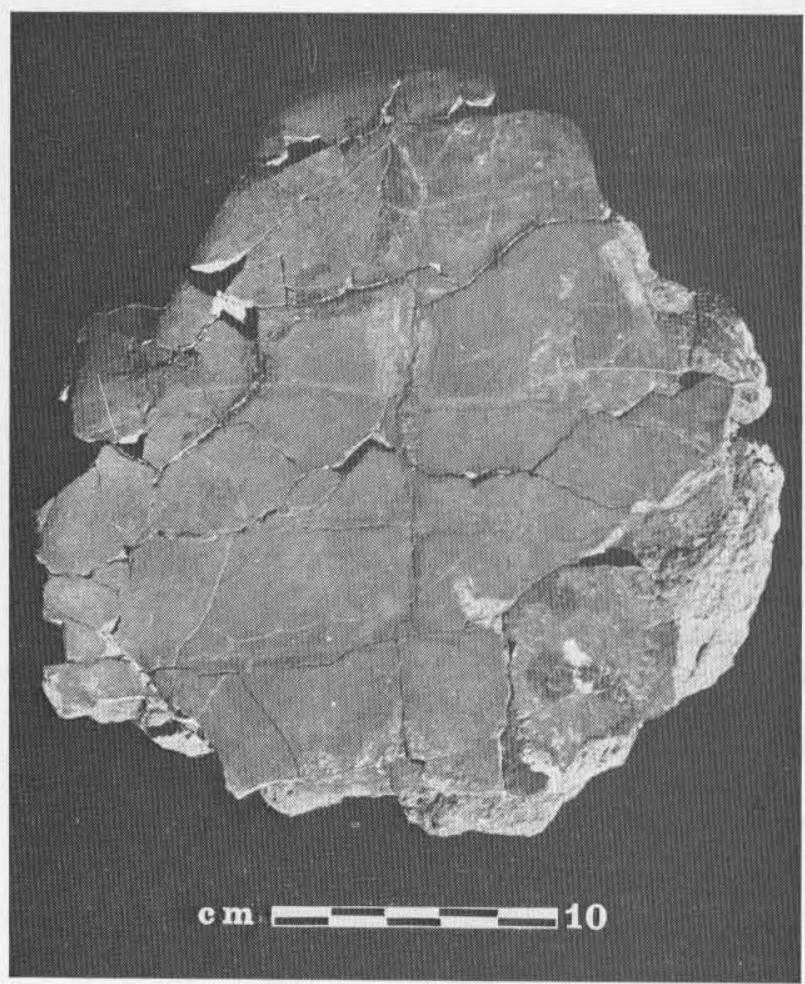

Fig. 2. - Duerochelys arribasi nov. gen., nov. sp. (Ex. 326, F. C. U. S.).

B.S.G.F., (7), XVII, 1975, no 3 . 
Les deux gulaires, triangulaires, ont aussi les sillons recourbés et leur forme ne se différencie pas de celle des Pelomedusidae du bassin du Duero. Ils parviennent à se superposer à l'entoplastron.

Les huméraux, trapézoïdaux, se touchent sur la ligne médiane. Le droit est plus allongé et effleure le pectoral gauche.

Le sillon pectoral-abdominal est droit et orthogonal à la ligne médiane sur une longueur de $50 \mathrm{~mm}$ mais il se recourbe ensuite à l'arrière comme cela se produisait pour le "Stereogenys" salmanticensis ; cependant celui-ci ne se superpose pas au mésoplastron. Même chose pour le sillon abdominal-fémoral, lequel se termine à l'aine exactement.

Plaques osseuses. L'entoplastron est d'aspect subrhomboïdal, aux côtés antérieurs droits et postérieurs légèrement ondulés. Sa face interne présente les proéminences typiques, ici en forme de trident, la branche centrale se prolongeant en avant.

Sur l'hyoplastron et s'inscrustant sur le moule détritique on peut voir la naissance des chambres sternales dont les supports ne sont pas excessivement résistants, comme c'était le cas pour le "Stereogenys " salmanticensis et le "Podocnemis" carbajosai (en ce point l'épaisseur est de $4,5 \mathrm{~mm}$ ).

La suture hyo-hypoplastrale est légèrement ondulée et ne rejoint pas la symétrique, de telle sorte que l'hyoplastron gauche atteint l'hypoplastron droit.

Les mésoplastrons sont petits et latéraux de forme mi-ovale, mi-pentagonale. Sur ceux-ci on distingue un sillon dermique marginal.

Les dimensions, en millimètres, sont : longueur du fragment 250 ; lobe antérieur : largeur 158 ; longueur 82 ; lobe postérieur : largeur 156 ; pont sternal : longueur 160.

\section{Écussons dermiques.}

Intergulaires : longueur 45 ; largeur 16 ; épaisseur 7,5 ; sillon médian 45 .

Gulaires : largeur 22 ; longueur 31,5 ; sillon avec les huméraux 37.

Huméraux : largeur 56 ; longueur 47 ; rebord 41 ; sillon avec pectoraux 58 ; sillon avec l'intergulaire 17 ; sillon interhuméraux 11,5 .

Pectoraux : largeur 112 ; longueur 75 ; sillon interpectoral 62 ; sillon avec l'abdominal 113.

Abdominaux : largeur 121 ; longueur 90 ; sillon interabdominal 80 ; sillon avec le fémoral 84 .

Fémoraux : largeur 76 ; longueur plus de 55 ; sillon interfémoral plus de 43 .

\section{Plaques osseuses.}

Entoplastron : largeur 72 ; longueur (L.) 59.

Épiplastrons : largeur 56 ; longueur 53 ; suture interépiplastrale 23 ; suture épi-hyoplastrale 26.

Hyoplastrons : largeur 108 ; longueur 117 ; suture interhyoplastrale 77 ; suture hyo-hypoplastrale 78 . Mésoplastrons : largeur 46 ; longueur 44 ; distance à l'axile 54 ; distance à l'aine 42 .

Hypoplastrons : largeur 78; longueur approx. 74 ; suture interhypoplastrale 74 ; suture hypoxiphiplastrale plus de 57.

Et en ce qui concerne la superposition des écussons épidermiques sur l'entoplastron [E. Jiménez, 1968] :

Sup. des intergulaires $\ldots \ldots \ldots \ldots$ du gulaire droit $\ldots \ldots \ldots \ldots$

" " gauche $\ldots \ldots \ldots$.

de l'huméral droit ...........

de "gauche ........

"l'interhuméral ...........

" de la suture interpectorale

(L) $\quad(x / \mathrm{L})$

$\overline{21,7} \quad \overline{0,37}$

$1,9 \quad 0,03$

$2,2 \quad 0,04$

$25,7 \quad 0,44$

$23,9 \quad 0,41$

$11,3 \quad 0,19$

$25,3 \quad 0,43$

demi-largeur $\ldots \ldots \ldots \ldots \ldots \ldots \ldots \quad 36,5 \quad 0,63$

Par l'observation de ces dimensions on peut constater qu'à l'exception des intergulaires, elles diffèrent fort peu de celles des autres Pelomedusidae du Duero.

PARATYPE : ex. 226 F.C.U.S. (fig. 3 et 4 )

Le spécimen en question est un entoplastron sur lequel se superpose une partie des deux intergulaires, gulaires, huméraux et pectoraux. Ses dimensions sont $29,5 \times 30,9 \mathrm{~mm}$. Il présente des sillons très curieux, moins profonds que les dermiques, dont nous ignorons toute signification.

Les dimensions en millimètres et pourcentages par rapport à la longueur de l'entoplastron sont :

\begin{tabular}{|c|c|c|c|}
\hline & & $(\mathrm{X})$ & $(x / \mathrm{L})$ \\
\hline Lon & queur & 29,5 & 00 \\
\hline Lar & eur $\ldots \ldots \ldots \ldots \ldots$ & 30,9 & 1, \\
\hline Sup & de l'intergulaire droit .... & 11,0 & \\
\hline$"$ & $" \quad " \quad$ gauche $\ldots$. & 10,1 & \\
\hline " & du gulaire droit $\ldots \ldots \ldots \ldots$ & 1,1 & 0,04 \\
\hline ” & $" \Rightarrow$ gauche $\ldots \ldots \ldots$ & $?$ & \\
\hline$"$ & de l'huméral droit .... & 15,6 & 0,5 \\
\hline$"$ & $" \quad " \quad$ gauche $\ldots . .$. & 12,1 & 0,41 \\
\hline$"$ & du sillon entre intergulaires. & 11,0 & 0,3 \\
\hline$"$ & $"$ " interhuméral $\ldots .$. & 6,4 & \\
\hline & interpectoral ..... & 12,1 & 0,41 \\
\hline
\end{tabular}

On peut remarquer que les dimensions de cet exemplaire sont quelque peu supérieures à la moitié de l'holotype. Néanmoins les mesures relatives se maintiennent très approximativernent constantes.

Sur sa face interne l'entoplastron présente l'insertion typique en forme de trident et deux paires d'orifices semblables aux deux autres Pelomedusidae du Duero [E. Jiménez, 1968 ; 1969 ; 1970 ; 1971 a ; $1971 \mathrm{c}]$. Les dimensions de ce côté-là sont légèrement 


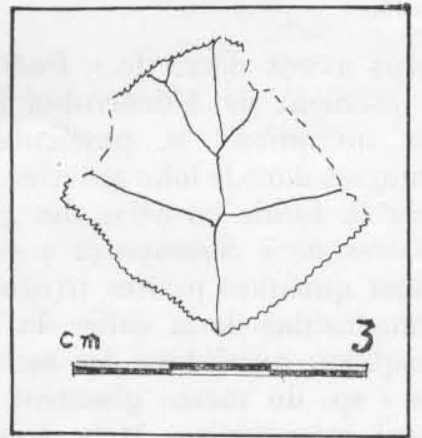

FIG. 3. - Duerochelys arribasi nov. gen., nov. sp. (Ex. 226, ti. F. C._U. S.). Schéma.

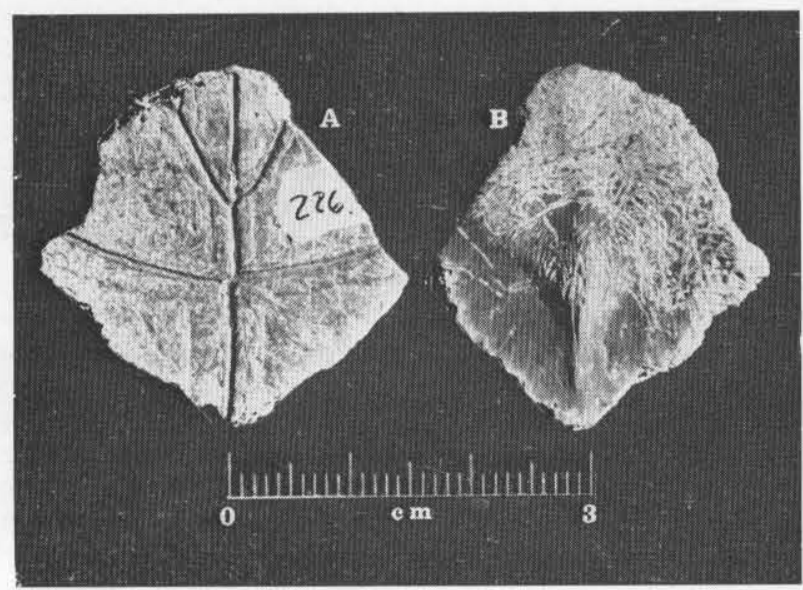

FIg. 4. - Duerochelys arribasi nov.' gen., 'nov. sp. (Ex. 226, 6. F. C. U. S.).

différentes : longueur $30,3 \mathrm{~mm}$; largeur 28,8. Peu d'épaisseur : $4 \mathrm{~mm}$ sur les côtés et 5,5 au centre.

Les différentes dimensions internes et externes sur une plaque si mince pourraient nous permettre de supposer que le spécimen correspond à une variété plus petite ou à une espèce différente, comme cela se produisait avec le "Podocnemis " entodermica [E. Jiménez, $1971 \mathrm{c}$ ], mais nous nous croyons insuffisamment informés pour avancer une telle affirmation.

\section{Rapports paléontologiques.}

Nous voulons insister une fois encore, sur ce fait, si ce n'était en raison de la présence de deux intergulaires, ces deux exemplaires ne se distingueraient pas des autres Pélomedusidae éocènes de la vallée du Duero. La présence des mésoplastrons justifie leur classification dans ladite famille [A. S. Romer, 1956].

La détermination générique de cette famille s'établit en se basant sur l'étude des crânes mais leur registre paléontologique est rare. Concrètement, jusqu'à présent, on n'en a pas encore trouvé en Europe. C'est pourquoi il a fallu attribuer les diverses carapaces de Chéloniens fossiles de cette famille soit à des genres actuels (principalement Podocnemis), soit à des genres nouveaux, si l'on se tient aux particularités morphologiques.

Il est très probable que l'attribution générique des "Podocnemis ") européens (et également de nos "Stereogenys ") subisse une modification essentielle avec la découverte des restes crâniens ce qui permettra de nouvelles lignes philétiques. Tout paraît indiquer que l'actuelle espèce malgache Erymnochelys madagascarensis GRAND. a beaucoup de rapports avec les anciennes espèces africaines [E. E. Williams, 1954], mais il serait prématuré d'établir des contacts entre celles-ci et leur parentes européennes. Une tentative semblable s'est récemment réalisée avec les rares formes asiatiques [R. C. Wood, 1970].

En ce qui concerne les deux exemplaires que nous décrivons dans ce travail, nous dirons qu'ils présentent une anomalie inédite dans cette famille. De récents travaux [E. S. Gaffney et R. Zangerl, 1968; R. Zangerl, 1969] démontrent que les variations dans la distribution des plaques osseuses et des écussons dermiques de la carapace n'ont pas de caractère générique. Un cas très spectaculaire se présente chez le Podocnemis venezuelensis [R. C. Wood, et M. L. Diaz de Gamero, 1971] avec absence totale de plaques neurales et chez les Pelomedusidae africains [F. M. Bergounioux, 1955, 1956 ; F. M. Bergounioux et F. Crouzel, 1968] décrits par Bergounioux comme "Eusarkiidae " et révisés récemment par Moody [1972]. D'autres anomalies en ce qui concerne les genres "Polysternum ", "Paralichelys » et "Asturichelys » [F. M. Bergouinoux, 1935, 1954, $1957 \mathrm{~b}$, 1958] présentent une disposition très confuse dont il conviendrait de revoir l'attribution de la famille. La distribution des plaques osseuses et des écussons dermiques du plastron des Pelomedusidae présente peu de variations d'une importance appréciable, celles-ci se rapportent principalement au plus ou moins grand développement de l'intergulaire, qui toujours est unique. L'exemplaire que nous décrivons est le premier qui présente deux intergulaires. Uniquement, le "Neochelys bolcensis " [F. M. Bergounioux, 1954] de Monte Bolca (Italie) peut avoir une certaine similitude.

Toujours dans la localité d'Aldearrubia, on a trouvé un autre fragment de Pelomedusidae dont la disposition des écussons dermiques dans le lobe antérieur du plastron est semblable à celles des espèces sud-américaines actuelles du genre Podocnemis. On a classé donc a priori cet exemplaire dans ce dernier genre, sans le spécifier. Ses gulaires nous montrent curieusement ùn aspect triangulaire élargi au sommet postérieur, et parviennent à se superposer à l'entoplastron. A la différence des deux exemplaires avec deux intergulaires que nous avons décrits ici, le "Podocnemis " sp. d'Aldearrubia 
montre clairement un seul intergulaire, qui, lui aussi, se superpose à l'entoplastron.

En raison de cette anomalie si particulière nous avons décidé de créer un genre nouveau que nous nommons DUEROCHELYS, toponymie du fleuve Duero, et que nous dédions spécialement à notre cher maître, ami et collègue M. Antonio Arribas, avec la diagnose suivante:

Classe : REPTILIA, Linné 1758

Ordre : Chelonia, Brongniart 1800

Famille : Polomedusidae, Cope 1868

Genre : DUEROCHELYS nov.

"Ses caractéristiques sont : il possède deux intergulaires symétriques et triangulaires, qui séparent complètement les gulaires et partiellement les huméraux, lesquels arrivent à se superposer à l'entoplastron. De petits mésoplastrons latéraux. "

Espèce unique : $D$. arribasi nov.

Gisements : sédiments détritiques fluviaux du Teso de las Canteras, de Aldearrubia (Salamanque, Espagne).

Age : Ludien.

Espèce-type et holotype : exemplaire 326 F.C.U.S.

Paratype : exemplaire 226 F.C.U.S.

\section{Conclusions.}

Lorsque nous avons décrit le "Podocnemis" sp. de ce même gisement de Aldearrubia [E. Jiménez, 1971d], nous indiquions la particularité de ses écussons dermiques dans le lobe antérieur du plastron manifestée par la forme curieuse des gulaires. Certains exemplaires de "Stereogenys " salmanticensis présentent aussi quelques petites irrégularités, mais tous les Pelomedusidae de la vallée du Duero qu'on a trouvés jusqu'ici, aussi bien les lutétiens que le "Podocnemis " sp. du même gisement ludien, possèdent un seul intergulaire. Mais pour le cas du Duerochelys arribasi nous pouvons parler de grandes anomalies : il semble que dans la vallée du Duero des changements sensibles se seraient produits quant à la structure dermique des Chéloniens pendant le passage du Lutétien au Ludien.

Les variations climatiques qui affectèrent le SE de l'Europe à partir de l'Oligocène durent être fatales pour ces Chéloniens. Ces variations eurentelles pour cause l'apparition d'anomalies chez les derniers représentants européens des Pelomedusidae ? Est-ce simplement un cas d'endémisme ?

Les renseignements que nous possédons actuellement sont insuffisants pour définir une théorie exacte.

1. Actuellement nous pensons que l'attribution aux genres Podocnemis et Stereogenys doit être considérée avec quelques réserves en attendant que la découverte de crânes permette de les classer comme des genres nouveaux.

\section{Bibliographie}

[1] Bergounioux F. M. (1935). --- Contribution à l'étude palćontologicque des Chéloniens : Chéloniens fossiles du Bassin d'Aquitaine. Mém. Soc. Géol. Fr., nouv. sér., t. XXV, p. 1-216, $16 \mathrm{pl}$.

[2] Bergocntoux F. M. (1954). - Chéloniens fossiles de la Vénétie. Mem. Ist. Geol. Miner. Univ. Padova, t. 18 , p. $1-116,16$ pl.

[3] Bergounioux F. M. (1955). - La famille des Eusarkiidés. C. R. Ac. Sc., Paris, t. 240, p. 1455-1457.

[4] Bergocniocx F. M. (1956). -... Les reptiles fossiles des dépôts phosphatés sud-tunisiens. Ann. Min. Géol. Tunis, Ł. 15, p. 1-103.

[5] Bergounioux F. M. (1957 b). -- Asturichelys multicostatus nov. gen., nov. sp. Monogr. Geol. Inst. Geol. Aplic. Oviedo, 4, p. 1-9.

[6] Bergounioux F.M. (1958). -- Les reptiles fossiles du Tertiaire de la Catalogne. Est. Geol., 14, p. 129219.

[7] Bergounioux F. M. et Crouzel F. (1968). .... Deux tortues fossiles d'Afrique. Bull. Soc. Hist. Nat. Toulouse, t. 104, no (1-2), p. 179-186.
[8] Chusafont M. et Truyols J. (1957). .-. Algunas precisiones sobre la edad y extension del paléggeno de las provincias de Salamanca y Zamora. Curs. y Conf. Inst. Lucas Mallada, no 4, p. 83-85.

[9] Gaffney E. S. et Zangerl R. (1968), - A revision of the genus Bothremys (Pleurodira, Pelomedusidae). Fieldiana Geol., 16, (7), p. 193-239.

[10] Herníndez Pacheco E. (1915). - Geologia y Paleontología del Mioceno de Palencia. Mem. Com. Invest. Paleont. y Prehist., 5, p. 1-295.

[11] Hernández Pacheco E. (1943). - - Observaciones respecto al Paleógeno continental hispánico. Las Ciencias, 8, (3), p. 455-555.

[12] JiMÉnez E. (1968). - Stereogenys salmanticensis nov. sp., quelonio eocénico del Valle del Duero. Est. Geol., 24, p. 191-203.

[13] JrmÉnEz E. (1969). - Sobre un nuevo hallazgo de Stereogenys salmanticensis Jim. Stvd. Geol., 1, p. 7386.

[14] Jiménez E. (1970). - Los reptiles fósiles del Valle del Duero : Sobre un cuarto paratipo casi completo

B.S.G.F., (7), XVII, 1975, no 3 . 
de Stereogenys salmanticensis Jıм. Est. Geol., 26, p. $245-260$.

[15] JimÉnez E. (1971 a). — Los reptiles fósiles del Valle del Duero : Podocnemis carbajosai nov. sp., del Eoceno de Salamanca. Est. Geol., 27, p. 85-93.

[16] Jiménez E. $(1971 b)$. — Primer Pseudotrionyx español : Allaeochelys casasecai nov, sp. ; del Luteciense de Corrales (Zamora). Est. Geol., 27, p. 153-166.

[17] JIMÉNEZ E. $(1971 c)$. - Nuevos fragmentos específicos de Pelomedúsidos lutecienses del Valle del Duero. Bol. R. Soc. Española Hist. Nat. (G), 68, p. 243-257.

[18] Jiménez E. $(1971 d)$. -- Un Podocnemis del Ludiense de Aldearrubia (Salamanca). Tomo Conm. Cent. R. Soc. Española Hist. Nat. (Geol.), (i. l.).

[19] Jiménez E. (1972). - El Paleógeno del borde SW de la Cuenca del Duero. I : Jos escarpes del Tormes. Stvd. Geol., 3, p. 67-110.

[20] JiméneZ E. (1973). - El Paleógeno del borde SW de la Cuenca del Duero. II : La falla de Alba-Villoria y sus implicaciones estratigráficas y geomorfologicas. Stod. Geol., 5, p. 107-136.

[21] Miquel M. (1906). - Restos fósiles de vertebrados encontrados en San Morales (Salamanca). Bol. R. Soc. Española Hist. Nat., 6, p. 352-357.

[22] Moody R. T. (1972). - The Turtle Fauna of the Eocene Phosphates of Metlaoui, Tunisia, Proc. Geol. Ass., 83, (3), p. 327-336, pl. 16-17.

[23] Puig Y Larraz G. (1883). —- Descripción física y geológica de la provincia de Zamora. Mem. Com. Mapa Geol. España, Madrid.
[24] Roman F. et Royo Gómez J. (1922). - Sur l'existence de Mammifères lutéciens dans le bassin du Douro (Espagne). C. R. Ac. Sc., Paris, t. 175, p. 1221-1223.

[25] Roman F. (1923). - Algunos dientes de Lofiodóntidos descubiertos en España. Mem. Com. Invest. Paleont. $y$ Prehist., 33, p. 1-22.

[26] Romer A. S. (1956). - Osteology of the Reptiles. The Univ. Ghicago Press; p. 1-772, 248 fig.

[27] Saenz C. (1934). - Nuevos yacimientos de vertebrados fósiles en la Cuenca Terciaria del Duero. Bol. $R$. Soc. Española Hist. Nat., 34, p. 181-185.

[28] Vilanova J. (1873). - Noticia de vertebrados hallados en Sanzoles (Zamora). Actas R. Soc. Española Hist. Nat., p. $42-47$ et 52 .

[29] Williams E. E. (1954). - New or redescribed Pelomedusid skulls from the Tertiary of Africa and Asia (Testudines, Pelomedusidae). 2. A Podocnemide skull from the Miocene of Moghara, Egypt. Breviora, 39, p. 1-10, pl. 1-2.

[30] Wood R. C. (1970), - A review of the fossil Pelomedusidae (Testudines, Pleurodira) of Asia. Breviora, n' 357, p. 1-24, pl. 1-4.

[31] Wood R. C. et Diaz de Gamero M. L. (1971). - Podocnemis venezuelensis, a new fossil Pelomedusid (Testudines, Pleurodira) from the Pllocene of Venezuela and a review of the history of Podocnemis in South America. Breviora, $\mathrm{n}^{\circ} 376$, p. 1-23, pl. 1-5.

[32] Zangerl R. (1969). - The Turtle Shell, In Biology of the Reptiles. V. 1. London, Ed. C. Gans, p. 311-340. 